\title{
Numerical Solution of Nonlinear Klein-Gordon Equation Using Lattice Boltzmann Method
}

\author{
Qiaojie Li ${ }^{1}$, Zong $\mathbf{J i}^{2}$, Zhoushun Zheng ${ }^{1}$, Hongjuan Liu ${ }^{1}$ \\ ${ }^{1}$ School of Mathematical Science and Computing Technology, Central South University, Changsha, China \\ ${ }^{2}$ Modern Service and Trade College, Yunnan University of Finance and Economics, Kunming, China \\ E-mail:qiaojie_li@foxmail.com \\ Received October 24, 2011; revised November 24, 2011; accepted December 1, 2011
}

\begin{abstract}
In this paper, in order to extend the lattice Boltzmann method to deal with more nonlinear equations, a onedimensional (1D) lattice Boltzmann scheme with an amending function for the nonlinear Klein-Gordon equation is proposed. With the Taylor and Chapman-Enskog expansion, the nonlinear Klein-Gordon equation is recovered correctly from the lattice Boltzmann equation. The method is applied on some test examples, and the numerical results have been compared with the analytical solutions or the numerical solutions reported in previous studies. The $L_{2}, L_{\infty}$ and Root-Mean-Square (RMS) errors in the solutions show the efficiency of the method computationally.
\end{abstract}

Keywords: Lattice Boltzmann, Chapman-Enskog Expansion, Nonlinear Klein-Gordon Equation

\section{Introduction}

Nonlinear phenomena modeled by partial differential equation appear in many areas of scientific fields such as solid state physics, plasma physics, fluid dynamics, mathematical biology and chemical kinetics. The nonlinear Klein-Gordon equation has attracted much attention in studying solutions and condensed mater physics, in investigating the interaction of solitons in a collisionless plasma, the recurrence of initial states, and in examining the nonlinear wave equations $[1,2]$. In the last few decades, many powerful methods, such as the inverse scattering method, Baklund transformation, the auxiliary equation method $[3,4]$, the Wadati trace method, Hirota bilinear forms, the tanh-sech method, the sine-cosine method, Jacobi elliptic functions, and the Riccati equation expansion method were used to investigate these types of equations (see [5] and references therein). A variety of finite difference scheme have been presented (see [6] and references therein) and the alternative approaches using spectral and pseudo-spectral methods have recently been presented $[7,8]$. To avoid the mesh generation, meshless techniques have attracted the attention of researchers in recent years. The radial basis function (RBF) as a truly meshless method was used to solve nonlinear Klein-Gordon equation in [9].

Recently, unlike convectional numerical methods which search for the macroscopic equation, the lattice Boltzmann method (LBM) has achieved much success in studying nonlinear equations and the evolution of complex systems $[10,11]$. By choosing appropriate collision or equilibrium distribution, the lattice Boltzmann model is able to recover the PDE of interest. This method is a new technique based on a mesoscopic kinetic equation for the particle distribution functions. Compared with the conventional numerical methods, the LBM provides many of the advantages, including geometrical flexibility, clear physical pictures, ease in incorporating complex boundary conditions, simplicity of programming and numerical efficiency. Recently, it has been developed to simulate linear and nonlinear PDE such as Laplace equation [12], Poisson equation [13,14], the shallow water equation [15], Burgers equation [16], Korteweg-de Vires equation [17], Wave equation [18,19], reaction-diffusion equation [20,21], convection-diffusion equation [22-24].

In this paper, the initial-value problem of the one-dimensional nonlinear Klein-Gordon equations is given by the following equation,

$$
u_{t t}+\alpha u_{x x}+g(u)=f(x, t)
$$

where $u=u(x, t)$ represents the wave displacement at position $x$ and time $t, \alpha$ is a known constant and $g(u)$ is the nonlinear force.

The present work is motivated by the desire to extend 
the lattice Boltzmann method to deal with evolution models characterized by nonlinear wave dispersion. By using Taylor expansion and the Chapman-Enskog expansion, the second-order nonlinear Klein-Gordon equation can be recovered from the present model correctly. The local equilibrium distribution function and the amending function are obtained. To make a comparison between numerical solutions and analytical ones, four Klein-Gordon equations with quadratic or cubic nonlinearity are considered. From the simulations, we find that the numerical results are in excellent agreement with the analytical solutions. This indicates that the present method is an efficient and flexible approach for practical application.

The organization of the paper as follows. In Section 2, the lattice Boltzmann model is described. Numerical examples are simulated in Section 3. Summary and conclusion are presented in Section 4.

\section{The Lattice Boltzmann Model}

The lattice Boltzmann model used on this study is the three-velocity lattice Bhatnagar-Gross-Krook (LBGK) model. The directions of the discrete velocity are defined as $c_{i} \quad(i=0,1,2)$

$$
\left[c_{0}, c_{1}, c_{2}\right]=[0,-c, c] \text {. }
$$

where $c$ is a constant. The lattice Boltzmann equation with an amending function is given as follow

$$
\begin{aligned}
& f_{i}\left(x+c_{i} \Delta t, t+\Delta t\right)-f_{i}(x, t) \\
& =-\frac{1}{\tau}\left[f_{i}(x, t)-f_{i}^{e q}(x, t)\right]+\Delta t F_{i}(x, t)
\end{aligned}
$$

where $f_{i}(x, t)$ and $f_{i}^{e q}(x, t)$ are defined as the distribution and equilibrium distribution function, respectively. $F_{i}(x, t)$ is an amending function and $\tau$ is the dimensionless relaxation time. $\Delta x=c_{i} \Delta t$ and $\Delta t$ are the lattice spacing and time step, respectively.

Unlike for the normal LBM, the first derivative of the macroscopic variable $u(x, t)$ meets the following conservation laws

$$
\sum_{i} f_{i}(x, t)=\sum_{i} f_{i}^{e q}(x, t)=\frac{\partial u(x, t)}{\partial t}
$$

Then, through choosing appropriate local equilibrium distributions, we can retrieve the corresponding macroscopic equation correctly.

Indeed, applying the Taylor expansion to left-hand side of Equation (2) and retaining terms up to $\mathcal{O}\left(\Delta t^{3}\right)$, we get

$$
\begin{gathered}
\Delta t\left(c_{i} \frac{\partial}{\partial x}+\frac{\partial}{\partial t}\right) f_{i}+\frac{\Delta t^{2}}{2}\left(c_{i} \frac{\partial}{\partial x}+\frac{\partial}{\partial t}\right)^{2} f_{i} \\
+\mathcal{O}\left(\Delta t^{3}\right)=-\frac{1}{\tau}\left(f_{i}-f_{i}^{e q}\right)+\Delta t F_{i}
\end{gathered}
$$

The macroscopic equation can be recovered in the multi-scale analysis using a small expansion parameter $\varepsilon$ which is proportional to the ration of the lattice spacing to the characteristic macroscopic length. To do this, the Chapman-Enskog expansion in time and space is applied:

$$
\begin{aligned}
& f_{i}=f_{i}^{e q}+\varepsilon f_{i}^{(1)}+\varepsilon^{2} f_{i}^{(2)}, F_{i}=\varepsilon^{2} F_{i}^{(2)} \\
& \frac{\partial}{\partial t}=\varepsilon \frac{\partial}{\partial t_{1}}+\varepsilon^{2} \frac{\partial}{\partial t_{2}}, \frac{\partial}{\partial x}=\varepsilon \frac{\partial}{\partial x_{1}}
\end{aligned}
$$

where $f_{i}^{(k)}$ and $F_{i}^{(2)}$ are the non-equilibrium distribution functions and non-equilibrium amending function, which satisfy the solvability conditions

$$
\begin{aligned}
& \sum_{i} f_{i}^{(k)}=0(k \geq 1) \\
& \sum_{i} F_{i}^{(2)}=F^{(2)}
\end{aligned}
$$

Substituting Equation (5) into (4), we have

$$
\begin{aligned}
& \left(\varepsilon c_{i} \frac{\partial}{\partial x_{1}}+\varepsilon \frac{\partial}{\partial t_{1}}+\varepsilon^{2} \frac{\partial}{\partial t_{2}}\right)\left(f_{i}^{e q}+\varepsilon f_{i}^{(1)}+\varepsilon^{2} f_{i}^{(2)}\right) \\
& +\frac{\Delta t}{2}\left(\varepsilon c_{i} \frac{\partial}{\partial x_{1}}+\varepsilon \frac{\partial}{\partial t_{1}}+\varepsilon^{2} \frac{\partial}{\partial t_{2}}\right)^{2}\left(f_{i}^{e q}+\varepsilon f_{i}^{(1)}+\varepsilon^{2} f_{i}^{(2)}\right)(7) \\
& =-\frac{1}{\tau}\left(\varepsilon f_{i}^{(1)}+\varepsilon^{2} f_{i}^{(2)}\right)+\varepsilon^{2} F_{i}^{(2)}
\end{aligned}
$$

Comparing the two sides of Equation (7) and treating terms in order of $\varepsilon$ and $\varepsilon^{2}$ gives

$$
\begin{aligned}
& \mathcal{O}(\varepsilon):\left(c_{i} \frac{\partial}{\partial x_{1}}+\frac{\partial}{\partial t_{1}}\right) f_{i}^{e q}=-\frac{1}{\tau \Delta t} f_{i}^{(1)} \\
& \mathcal{O}\left(\varepsilon^{2}\right): \quad \frac{\partial}{\partial t_{2}} f_{i}^{e q}+\left(c_{i} \frac{\partial}{\partial x_{1}}+\frac{\partial}{\partial t_{1}}\right) f_{i}^{(1)} \\
& +\frac{\Delta t}{2}\left(c_{i} \frac{\partial}{\partial x_{1}}+\frac{\partial}{\partial t_{1}}\right)^{2} f_{i}^{e q}=-\frac{1}{\tau \Delta t} f_{i}^{(2)}+F_{i}^{(2)}
\end{aligned}
$$

Applying Equation (8) to the left side of Equation (9), we can rewrite Equation (9) as

$$
\begin{array}{r}
\frac{\partial}{\partial t_{2}} f_{i}^{e q}+\left(1-\frac{1}{2 \tau}\right)\left(c_{i} \frac{\partial}{\partial x_{1}}+\frac{\partial}{\partial t_{1}}\right) f_{i}^{(1)} \\
=-\frac{1}{\tau \Delta t} f_{i}^{(2)}+F_{i}^{(2)}
\end{array}
$$

In order to recover Equation (1), we must give appropriate local equilibrium distribution function. We choose $f_{i}^{e q}$ such that,

$$
\begin{gathered}
\sum_{i} f_{i}^{e q}=\frac{\partial u(x, t)}{\partial t} \\
\sum_{i} c_{i} f_{i}^{e q}=0, \sum_{i} c_{i} c_{i} f_{i}^{e q}=c_{s}^{2} u(x, t)
\end{gathered}
$$


where $c_{s}^{2}=c^{2} / 3$ is called the lattice Boltzmann sound speed. Equation (11) leads to three linear equations for $f_{i}^{e q}(x, t)$. Solving these equations determines the equilibrium distribution functions

$$
\begin{aligned}
f_{0}^{e q}(x, t) & =\frac{\partial u(x, t)}{\partial t}-\frac{u(x, t)}{3} \\
f_{1}^{e q}(x, t) & =\frac{u(x, t)}{6} \\
f_{2}^{e q}(x, t) & =\frac{u(x, t)}{6}
\end{aligned}
$$

Meanwhile, the amending function $F_{i}(x, t)$ is taken as

$$
F_{i}(x, t)=\omega_{i} F(x, t)=\omega_{i}(f(x, t)-g(u))
$$

such that $\sum_{i} F_{i}(x, t)=F(x, t)$. For simplicity, only one case is given here

$$
\begin{aligned}
& F_{0}(x, t)=\frac{2}{3}(f(x, t)-g(u)) \\
& F_{1}(x, t)=\frac{1}{6}(f(x, t)-g(u)) \\
& F_{2}(x, t)=\frac{1}{6}(f(x, t)-g(u))
\end{aligned}
$$

Summing Equation (8) and Equation (10) over $i$, and using Equation (6) and (11), we obtain

$$
\begin{gathered}
\frac{\partial}{\partial t_{1}}\left(\frac{\partial u}{\partial t}\right)=0 \\
\frac{\partial}{\partial t_{2}}\left(\frac{\partial u}{\partial t}\right)+\left(1-\frac{1}{2 \tau}\right) \frac{\partial}{\partial x_{1}}\left(\sum_{i} c_{i} f_{i}^{(1)}\right)=F^{(2)}
\end{gathered}
$$

Using Equation (8) and (11), we get

$$
\begin{aligned}
& \sum_{i} c_{i} f_{i}^{(1)}=-\tau \Delta t \sum_{i} c_{i}\left(c_{i} \frac{\partial}{\partial x_{1}}+\frac{\partial}{\partial t_{1}}\right) f_{i}^{e q} \\
& =-\tau \Delta t \sum_{i}\left[\frac{\partial}{\partial x_{1}}\left(c_{i} c_{i} f_{i}^{e q}\right)+\frac{\partial}{\partial t_{1}}\left(c_{i} f_{i}^{e q}\right)\right] \\
& =-\tau \Delta t \frac{\partial}{\partial x_{1}}\left(c_{s}^{2} u\right)
\end{aligned}
$$
have

Then substituting Equation (17) into Equation (16), we

$$
\frac{\partial}{\partial t_{2}}\left(\frac{\partial u}{\partial t}\right)+c_{s}^{2} \Delta t\left(\frac{1}{2}-\tau\right) \frac{\partial}{\partial x_{1}}\left(\frac{\partial u}{\partial x_{1}}\right)=F^{(2)}
$$

When Equation $(15) \times \varepsilon+(18) \times \varepsilon^{2}$ is applied, the final nonlinear Klein-Gordon equation is recovered as

$$
\frac{\partial^{2} u}{\partial t^{2}}+\alpha \frac{\partial^{2} u}{\partial x^{2}}=F(x, t)=f(x, t)-g(u)
$$

where $\alpha=c_{s}^{2} \Delta t\left(\frac{1}{2}-\tau\right)$

In the computational process, in order to obtain $u(x, t)$, we can apply backward difference to the item $\frac{\partial u(x, t)}{\partial t}$

$$
\frac{\partial u(x, t)}{\partial t}=\frac{u(x, t)-u(x, t-\Delta t)}{\Delta t}
$$

\section{Numerical Simulation Results}

In this section, we present the result of our LBM numerical experiments for the relevant equations. In comparison with the analytical solutions and results derived by existing literature, the efficiency of proposed model is validated. The distribution function $f_{i}(x, t)$ is initialized with $f_{i}^{e q}(x, t)$ for all nodes at $t=0$. The macroscopic variable $u(x, t)$ is initialized by the initial condition and the non-equilibrium extrapolation scheme proposed by Guo [25] is used for boundary treatment. The following error norms are used to measure the accuracy

1) $L_{2}$-error

$$
L_{2}-\text { error }=\left(\sum_{i=1}^{n} e_{i}^{2}\right)^{\frac{1}{2}}
$$

2) $L_{\infty}$-error

$$
L_{\infty}-\text { error }=\operatorname{Max} e_{i}, \quad 1 \leq i \leq n
$$

3) The root mean square (RMS) error

$$
R M S-\text { error }=\left(\sum_{i=1}^{n} \frac{e_{i}^{2}}{n}\right)^{\frac{1}{2}}
$$

where $e_{i}=\left|u\left(x_{i}, t\right)-u^{\star}\left(x_{i}, t\right)\right|, u\left(x_{i}, t\right)$ and $u^{\star}\left(x_{i}, t\right)$ are the numerical solution and analytical one.

Example 1. The Klein-Gordon equation with quadratic nonlinearity in the interval $-1 \leq x \leq 1$

$$
u_{t t}-u_{x x}=-x \cos t+x^{2} \cos ^{2} t-u^{2}
$$

The initial conditions are given by

$$
u(x, 0)=x, u_{t}(x, 0)=0
$$

The exact solution is given in [9]

$$
u(x, t)=x \cos t
$$

We extract the boundary condition from the exact solution. In Table 1, the $L_{\infty}, L_{2}$ and RMS errors are obtained for $t=1,3,5,7,10$. The graph of analytical and LBM solution for $t=1$ and $t=10$ are given in Figure 1 and the space-time graph of the LBM solution is given in Figure 2.

Example 2. Consider the nonlinear Klein-Gordon equation with quadratic nonlinearity in interval $0 \leq x \leq 1$. 
Table 1. $L_{\infty}, L_{2}$ and RMS errors with $\mathrm{d} x=0.02$ and $\mathrm{d} t=2 \times$ $10^{-5}$.

\begin{tabular}{cccc}
\hline \multirow{2}{*}{$t$} & \multicolumn{3}{c}{ errors } \\
\cline { 2 - 4 } & $L_{\infty}$-error & $L_{2}$-error & RMS \\
\hline 1 & $1.9558 \mathrm{e}-03$ & $1.1135 \mathrm{e}-03$ & $1.1294 \mathrm{e}-04$ \\
3 & $1.3664 \mathrm{e}-03$ & $7.6676 \mathrm{e}-03$ & $7.6295 \mathrm{e}-04$ \\
5 & $1.5260 \mathrm{e}-03$ & $8.5602 \mathrm{e}-03$ & $8.5178 \mathrm{e}-04$ \\
7 & $1.6201 \mathrm{e}-03$ & $9.5926 \mathrm{e}-03$ & $9.5450 \mathrm{e}-04$ \\
10 & $1.0465 \mathrm{e}-03$ & $6.9848 \mathrm{e}-03$ & $6.9501 \mathrm{e}-04$ \\
\hline
\end{tabular}
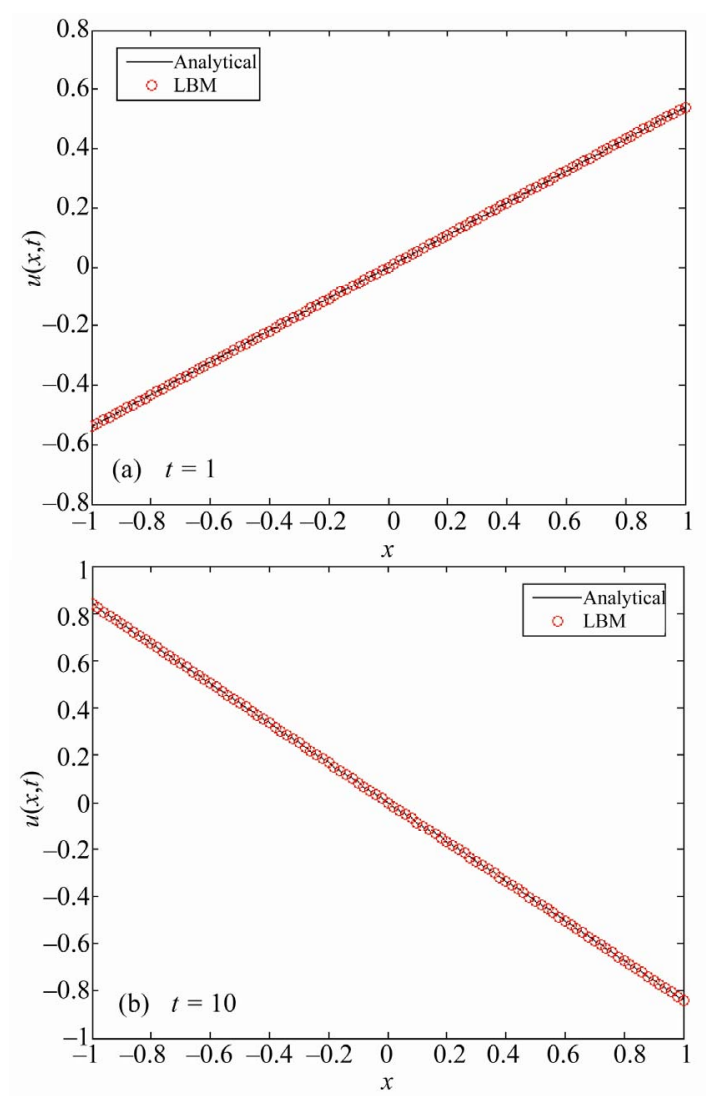

Figure 1. Analytical and LBM solutions with $\mathrm{d} x=0.02$ and $\mathrm{d} t=2 \times 10^{-5}$ for different time.

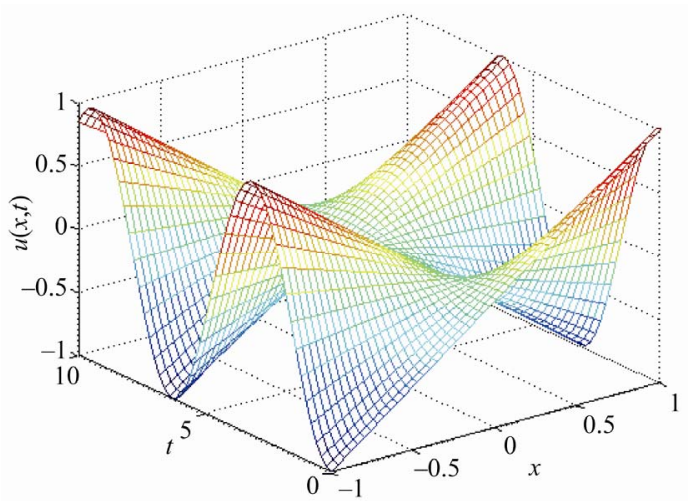

Figure 2. Space-time graph of the LBM solutions up to $t=$ 10 with $\mathrm{d} x=0.02$ and $\mathrm{d} t=2 \times 10^{-5}$.

$$
u_{t t}-u_{x x}=6 x t\left(x^{2}-t^{2}\right)+x^{6} t^{6}-u^{2}
$$

The initial conditions are given by

$$
u(x, 0)=0, u_{t}(x, 0)=0
$$

The exact solution is given in [9]

$$
u(x, t)=x^{3} t^{3}
$$

The Boundary condition is determined by the analytical solution. In Table 2, the $L_{\infty}, L_{2}$ and RMS errors are obtained for $t=1,2,3,4,5$. The graph of analytical and LBM solution for $t=5$ and the space-time graph of the LBM solution are given in Figure 3 and Figure 4 , respectively.

Example 3. The nonlinear Klein-Gordon equation with cubic nonlinearity in interval $-1 \leq x \leq 1$.

$$
u_{t t}+\alpha u_{x x}=-\beta u-\gamma u^{3}
$$

We take $\alpha=-2.5, \beta=1, \gamma=1.5$ as the same in [9]. The initial conditions are given by

$$
u(x, 0)=B \tan (K x), u_{t}(x, 0)=B c K \sec ^{2}(K x)
$$

The exact solution is

$$
u(x, t)=B \tan (K(x+c t))
$$

where $B=\sqrt{\beta / \gamma}$ and $K=\sqrt{-\beta / 2\left(\alpha+c^{2}\right)}$. In Table 3 , the $L_{\infty}, L_{2}$ and RMS errors are obtained for two values of $c(c=0.5$ and $c=0.05)$ for $t=1,2,3,4$. The graph of analytical and LBM solution for $t=4$ and the space-time graph of the LBM solution for each value of $c$ are given in Figure 5 and Figure 6, respectively.

Example 4. We consider the nonlinear Klein-Gordon equation with the form [9].

$$
u_{t t}-u_{x x}=-u-u^{3} ; x \in[0,1.28]
$$

with initial data

$$
u(x, 0)=A\left[1+\cos \left(\frac{2 \pi x}{1.28}\right)\right], u_{t}(x, 0)=0
$$

The boundary conditions are given by

$$
u_{x}(0, t)=0, u_{x}(1.28, t)=0
$$

Table 2. $L_{\infty}, L_{2}$ and RMS errors with $\mathrm{d} x=0.01$ and $\mathrm{d} t=5 \times$ $10^{-5}$.

\begin{tabular}{cccc}
\hline \multirow{2}{*}{$t$} & \multicolumn{3}{c}{ errors } \\
\cline { 2 - 4 } & $L_{\infty}$-error & $L_{2}$-error & RMS \\
\hline 1 & $5.8742 \mathrm{e}-04$ & $1.9270 \mathrm{e}-03$ & $1.9174 \mathrm{e}-04$ \\
2 & $4.6618 \mathrm{e}-03$ & $2.1643 \mathrm{e}-02$ & $2.1535 \mathrm{e}-03$ \\
3 & $1.5139 \mathrm{e}-02$ & $4.9465 \mathrm{e}-02$ & $4.9219 \mathrm{e}-03$ \\
4 & $3.4225 \mathrm{e}-02$ & $8.5102 \mathrm{e}-02$ & $8.4679 \mathrm{e}-03$ \\
5 & $6.3219 \mathrm{e}-02$ & $9.3035 \mathrm{e}-02$ & $1.2970 \mathrm{e}-02$ \\
\hline
\end{tabular}


Table 3. $L_{\infty}, L_{2}$ and RMS errors with $\mathrm{d} x=0.01$ and $\mathrm{d} t=5 \times$ $10^{-5}$.

\begin{tabular}{cccc}
\hline \multirow{2}{*}{$t$} & \multicolumn{3}{c}{ errors } \\
\cline { 2 - 4 } & $L_{\infty}$-error & $L_{2}$-error & RMS \\
\hline$c=0.5$ & & & \\
1 & $1.4189 \mathrm{e}-04$ & $6.6508 \mathrm{e}-04$ & $6.6171 \mathrm{e}-05$ \\
3 & $4.6601 \mathrm{e}-04$ & $1.5438 \mathrm{e}-03$ & $1.5362 \mathrm{e}-04$ \\
3 & $1.9445 \mathrm{e}-03$ & $4.9588 \mathrm{e}-03$ & $4.9342 \mathrm{e}-04$ \\
4 & $2.8219 \mathrm{e}-02$ & $7.1870 \mathrm{e}-02$ & $7.1513 \mathrm{e}-03$ \\
$c=0.05$ & & & \\
1 & $5.6970 \mathrm{e}-05$ & $2.9718 \mathrm{e}-04$ & $2.9570 \mathrm{e}-05$ \\
2 & $7.4878 \mathrm{e}-05$ & $3.8699 \mathrm{e}-04$ & $3.8507 \mathrm{e}-05$ \\
3 & $1.1972 \mathrm{e}-04$ & $5.2203 \mathrm{e}-04$ & $5.1944 \mathrm{e}-05$ \\
4 & $1.4008 \mathrm{e}-04$ & $4.4143 \mathrm{e}-04$ & $4.3924 \mathrm{e}-05$ \\
\hline
\end{tabular}

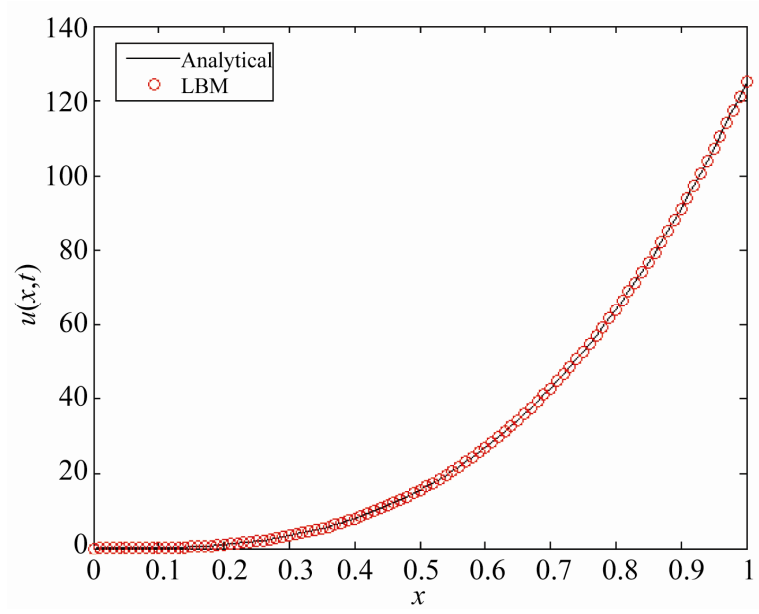

Figure 3. Analytical and LBM solutions at $t=5$ with $\mathrm{d} x=$ 0.01 and $\mathrm{d} t=5 \times 10^{-5}$.

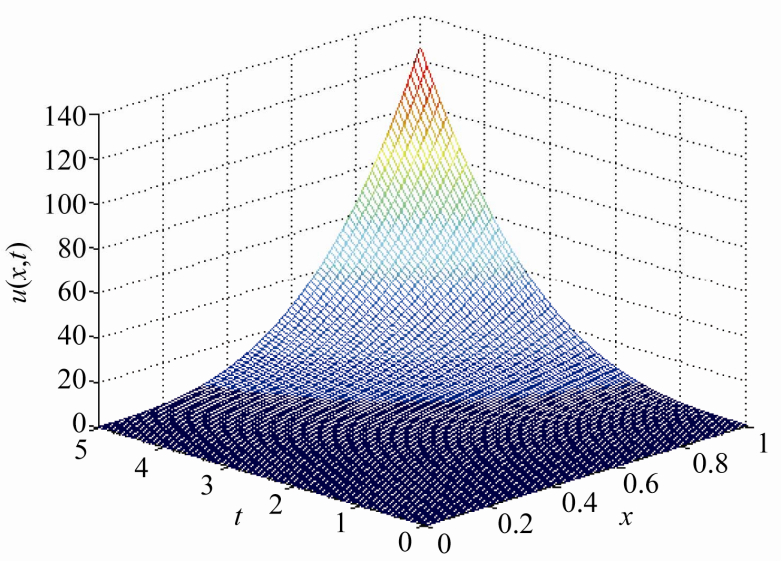

Figure 4. Space-time graph of the $\mathrm{LBM}$ solutions up to $t=5$ with $\mathrm{d} x=0.01$ and $\mathrm{d} t=5 \times 10^{-5}$.
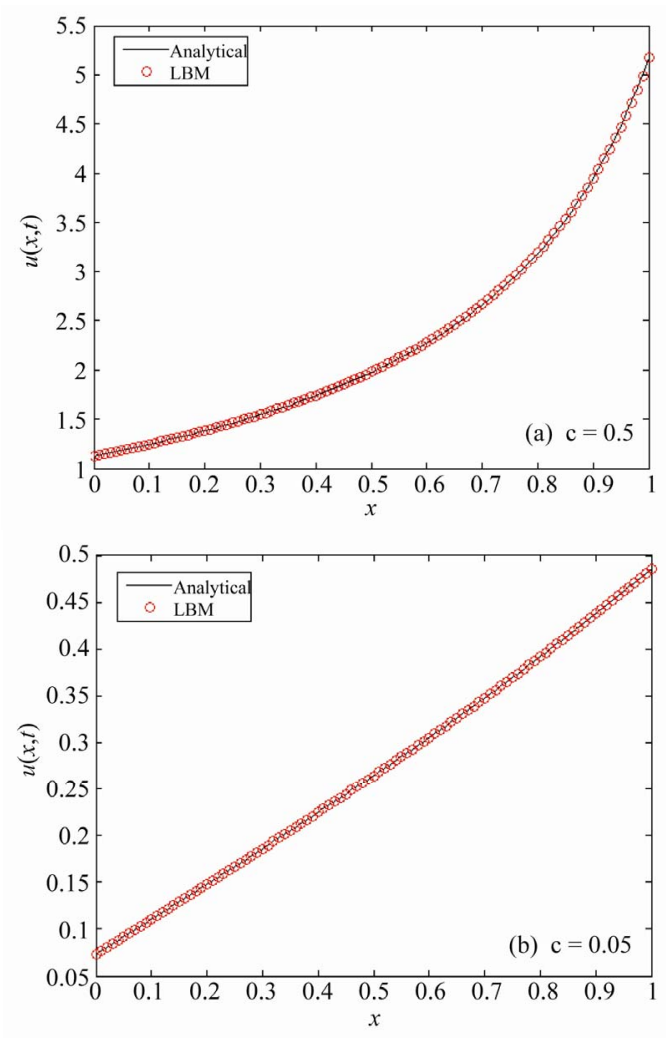

Figure 5. Analytical and LBM solutions at $t=4$ with $\mathrm{d} x=$ 0.01 and $\mathrm{d} t=5 \times 10^{-5}$ for different $c$.
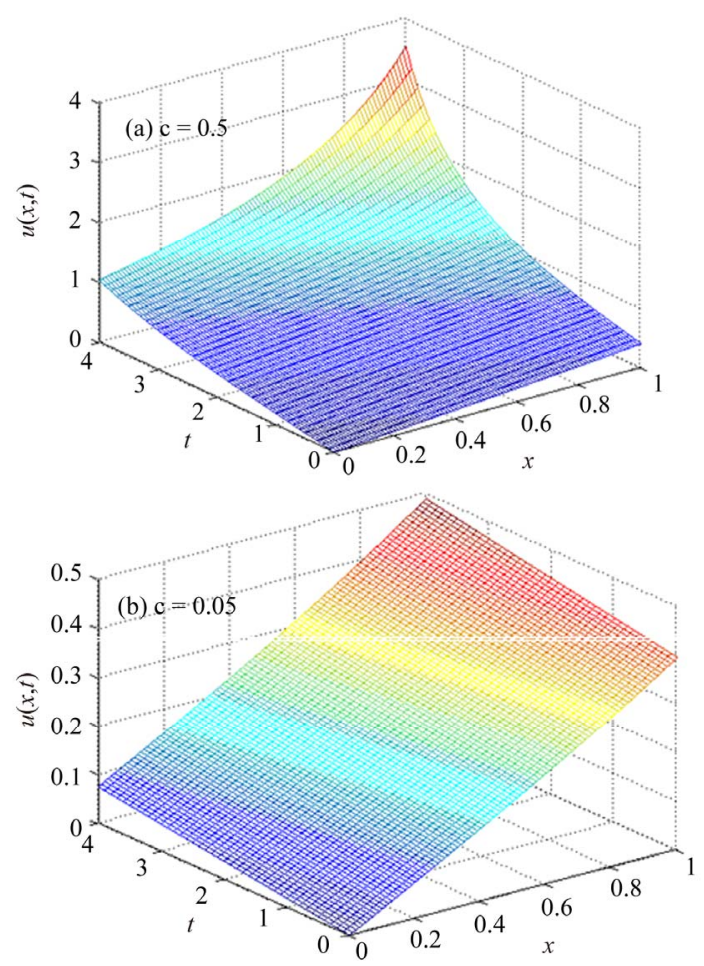

Figure 6. Space-time graph of the LBM solutions up to $t=4$ with $\mathrm{d} x=0.01$ and $\mathrm{d} t=5 \times 10^{-5}$ for different $c$. 
For the above problem due to the periodic boundary conditions, the continuous solutions remain always symmetric with respect to the center of the spatial interval. Authors of [26] also studied this problem and found undesirable characteristics in some of the numerical schemes, in particular a loss of spatial symmetry and the onset of instability for larger values of the parameter A (amplitude) in the initial condition of the equation. We solved the above problem using lattice Boltzmann method for several values of $A$. In Figure 7, we show the approximate solutions for $A=1$ with $\Delta x=0.0128$ and $\Delta t=1.8286 \times 10^{-5}$. Figure 8 presents the approximate solutions for $A=100$ with $\Delta x=0.0128$ and $\Delta t=1.8286 \times 10^{-5}$. From Figure 7 and Figure 8, we can find that the spatial symmetry is kept for different amplitude $A$. It indicates that the present lattice Boltzmann method is comparable with other numerical schemes.

\section{Conclusions}

In the current study, a new lattice Boltzmann model is proposed to solve 1D nonlinear Klein-Gordon equation. The efficiency and accuracy of the proposed model are validated through detail numerical simulation with quadratic and cubic nonlinearity. It can be found that the LBGK results are in excellent agreement with the analytical solution. It should be point out that in order to attain better accuracy the lattice Boltzmann model requires a relatively small time step $\Delta t$ and the proper range is form $10^{-4}$ to $10^{-6}$. Detailed stability analysis of present model is needed in further study.

\section{Acknowledgements}

This work was supported by the National Natural Sci-

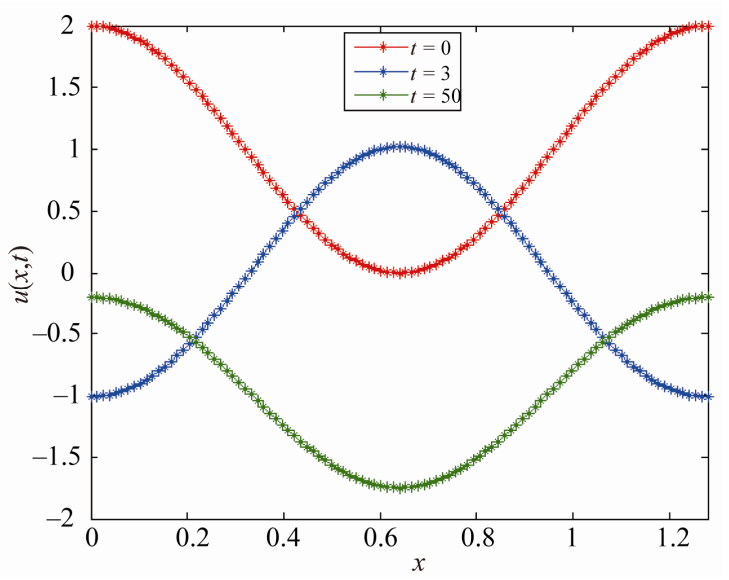

Figure 7. $\mathrm{LBM}$ solution at $t=0,3,50$ with $A=1, \mathrm{~d} x=$ 0.0128 and $\mathrm{d} t=1.8286 \times 10^{-5}$.
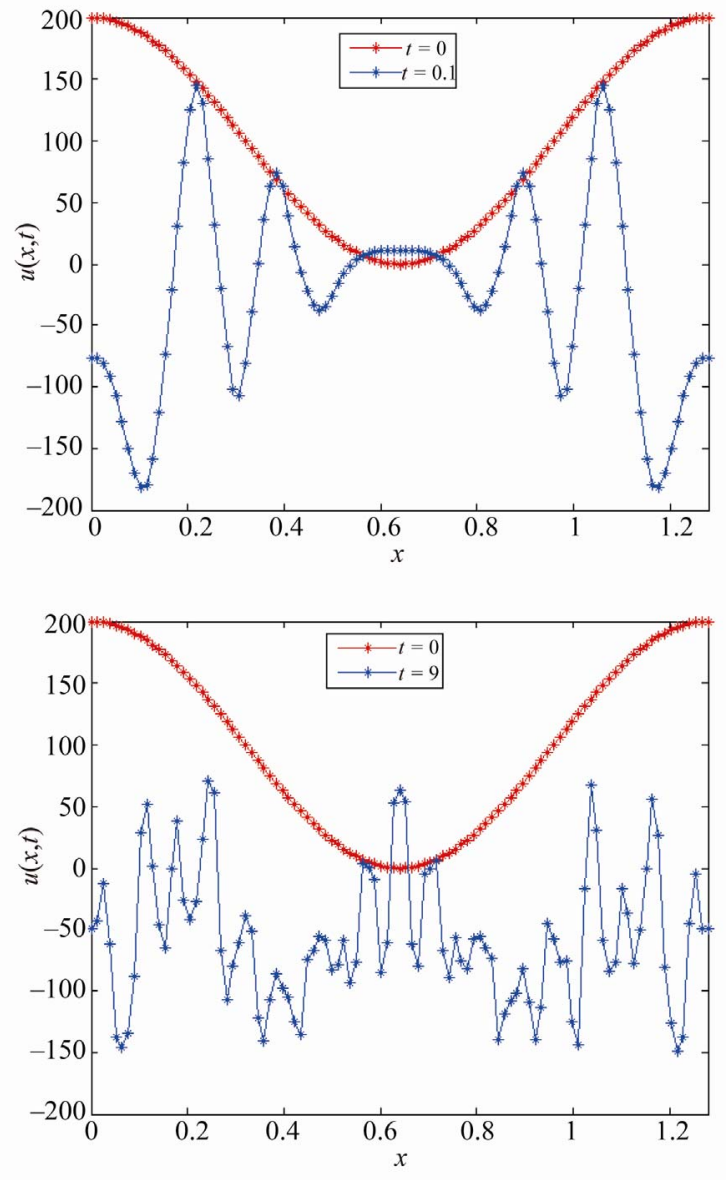

Figure 8. $\mathrm{LBM}$ solution with $A=100$ at different time $t$.

ence Foundation of China (50874123, 51174236) and National Basic Research Program of China (2011CB606306).

\section{References}

[1] P. J. Caudrey, I. C. Eilbeck and J. D. Gibbon, "The SineGordon Equation as a Model Classical Field Theory," Nuovo Cimento, Vol. 25, No. 2, 1975, pp. 497-511.

[2] R. K. Dodd, I. C. Eilbeck, J. D. Gibbon and H. C. Morris, "Solitons and Nonlinear Wave Equations," Academic, London, 1982.

[3] Sirendaoreji, "Auxiliary Equation Method and New Solutions of Klein-Gordon Equations," Chaos, Solitons \& Fractals, Vol. 31, No. 4, 2007, pp. 943-950. doi:10.1016/j.chaos.2005.10.048

[4] Sirendaoreji, "A New Auxiliary Equation and Exact Travelling Wave Solutions of Nonlinear Equation," Physics Letters A, Vol. 356, No. 2, 2006, pp. 124-130. doi:10.1016/i.physleta.2006.03.034

[5] A. M. Wazwaz, "New Travelling Wave Solutions to the Boussinesq and Klein-Gordon Equations," Communications in Nonlinear Science and Numerical Simulation, 
Vol. 13, No. 5, 2008, pp. 889-901. doi:10.1016/j.cnsns.2006.08.005

[6] M. A. Lynch, "Large Amplitude Instability in Finite Difference Approximates to the Klein-Gordon Equation," Applied Numerical Mathematics, Vol. 31, No. 2, 1999, pp. 173-182. doi:10.1016/S0168-9274(98)00128-7

[7] X. Li, B. Y. Guo and L. Vazquez, "A Legendre Spectral Method for Solving the Nonlinear Klein-Gordon Equation," Mathematics Applied and Computation, Vol. 15, No. 1, 1996, pp. 19-36.

[8] X. Li and B. Y. Guo, "A Legendre Spectral Method for Solving Nonlinear Klein-Gordon Equation," Journal Computation of Mathematics, Vol. 15, No. 2, 1997, pp. 105-126.

[9] M. Deghan and A. Shokri, "Numerical Solution of the Nonlinear Klein-Gordon Equation Using Radial Basis Functions," Journal of Computational and Applied Mathematics, Vol. 230, No. 2, 2009, pp. 400-410. doi:10.1016/j.cam.2008.12.011

[10] R. Benzi, S. Succi and M. Vergassola, "The Lattice Boltzmann Equation: Theory and Application," Physics Reports, Vol. 222, No. 3, 1992, pp. 145-197. doi:10.1016/0370-1573(92)90090-M

[11] S. Y. Chen and G. D. Doolen, "Lattice Boltzmann Method for Fluid Flows," Annual Review of Fluid Mechanics, Vol. 30, No. 1, 1997, pp. 329-364. doi:10.1146/annurev.fluid.30.1.329

[12] J. Y. Zhang, G. W. Yan and Y. F. Dong, "A New Lattice Boltzmann Model for the Laplace Equation," Applied Mathematics and Computation, Vol. 215, No. 2, 2009, pp. 539-547.doi:10.1016/j.amc.2009.05.047

[13] Z. H. Chai and B. C. Shi, "A Novel Lattice Boltzmann Model for the Poisson Equation," Applied Mathematical Modelling, Vol. 32, No. 10, 2008, pp. 2050-2058. doi:10.1016/j.apm.2007.06.033

[14] M. Hirabayashi, Y. Chen and H. Ohashi, "The Lattice BGK Model for the Poisson Equation," JSME International Journal Series B, Vol. 44, No. 1, 2001, pp. 45-52. doi:10.1299/jsmeb.44.45

[15] J. G. Zhou, "Lattice Boltzmann Method for Shallow Water Flows," Springer Verlag, New York, 2004.

[16] Z. Shen, G. Yuan and L. Shen, "Lattice Boltzmann Method for Burgers Equation," Chinese Journal of Com- putational Physics, Vol. 175, No. 1, 2000, pp. 172-177.

[17] J. Y. Zhang and G. W. Yan, "A Lattice Boltzmann Model for the Korteweg-de Vries Equation with Two Conservation Laws," Computer Physics Communications, Vol. 180, No. 7, 2009, pp. 1054-1062. doi:10.1016/j.cpc.2008.12.027

[18] G. W. Yan, "A Lattice Boltzmann Equation for Waves," Journal of Computational Physics, Vol. 161, No. 1, 2000, pp. 61-69. doi:10.1006/jcph.2000.6486

[19] J. Y. Zhang, G. W. Yan and X. Shi, "Lattice Boltzmann Model for Wave Propagation," Physics Review E, Vol. 80, 2009, Article ID 026706. doi:10.1103/PhysRevE.80.026706

[20] S. P. Dawson, S. Chen and G. D. Doolen, "Lattice Boltzmann Computations for Reaction-Diffusion Equation," Journal of Chemical Physics, Vol. 98, No. 2, 1993, pp. 1514-1523. doi:10.1063/1.464316

[21] X. Yu and B. C. Shi, "A Lattice Boltzmann Model for Reaction Dynamical Systems with Time Delay," Applied Mathematics and Computation, Vol. 181, No. 2, 2006, pp. 958-965. doi:10.1016/j.amc.2006.02.020

[22] S. R. Vander and M. Ernst, "Convection-Diffusion Lattice Boltzmann Scheme for Irregular Lattice," Journal of Computational Physics, Vol. 160, No. 2, 2000, pp. 766782. doi:10.1006/jcph.2000.6491

[23] Z. L. Guo, B. C. Shi and N. C. Wang, "Fully Lagrangian and Lattice Boltzmann Method for the Advection-Diffusion Equation," Journal of Scientific Computing, Vol. 14, No. 3, 1999, pp. 291-300. doi:10.1023/A:1023273603637

[24] B. C. Shi and Z. L. Guo, "Lattice Boltzmann Model for Nonlinear Convection-Diffusion Equations," Physics Review E, Vol. 79, 2009, Article ID 016701. doi:10.1103/PhysRevE.79.016701

[25] Z. L. Guo, C. G. Zheng and B. C. Shi, "Non-Equilibrium Extrapolation Method for Velocity and Pressure Boundary Conditions in the Lattice Boltzmann Method," Chinese Physics, Vo. 11, No. 4, 2002, pp. 366-374. doi:10.1088/1009-1963/11/4/310

[26] S. Jiminez and L. Vazquez, "Analysis of Four Numerical Scheme for a Nonlinear Klein-Gordon Equation," $A p$ plied Mathematics and Computation, Vol. 35, No. 1, 1990, pp. 61-94. doi:10.1016/0096-3003(90)90091-G 